\title{
Replaced ASCII table to encode-decode secret messages
}

\author{
Hatim Ghazi Zaini \\ Department of computer Engineering, Taif University, Taif, KSA
}

\begin{abstract}
Messages are circulated very widely through social media, and some of these messages may be confidential or have a personal character, which requires protection of this type of messages from people who are intrusive or have nothing to do with the message. In this paper, we will discuss a new method for encoding short and long text messages, which depends on the use of a special table modified for ASCII table, which includes the locations of the different symbols in a specific image. The proposed method is based on the use of the modified table, noting that 'this schedule changes from one image to another, which makes the process of understanding the encrypted text message impossible. The proposed method will be implemented and tested, and the obtained experimental results will be evaluated to show the efficiency and reliability of the proposed method.
\end{abstract}

Key words: Digital image, secret message, ASCII table, Updated table, encryption, decryption, throughput, efficiency, reliability.

\section{I- INTRODUCTION}

A short or long text message [4] is a set of symbols represented by ASCII table with fixed and specific values [17], [18] that are circulated between two people through social media. The text message may [31], [32] be of a personal nature as it contains personal information or it may contain confidential information that no third party has the right to view or hack into [1], [2], [3].

Currently [19], [20], many methods are used to encrypt-decrypt text messages, and some of these methods are based on international standards DES and AES and some of them depend on the exclusion or multiplication process [4]. [5]. [6]. Color digital images [7], [8] are one of the most prevalent types of data due to the many vital applications [9], [10] that use this group of data for several reasons, the most important of which are:

* $\quad$ Ease of getting the digital image at no cost [11], [12].

\# $\quad$ Ease of processing [13], [14].

\# High resolution, which provides a huge volume of data which can be used for multiple purposes [15], [16].

The digital color image is represented by a three-dimensional matrix [21], [22], where each of the three dimensions is assigned to a color (the first for red, the second for green, and the third for blue) [23], [24]. The point values in each color matrix range from 0 to 255 and these values cover the values in the ASCII table used to encode the different symbols that can constitute the secret message.

Here in this paper research we will introduce a new approach of message securing by using that uses the locations of the values between 0 and 255 to form a new updated table, which can be used to re-encode the secret message and generate the encrypted secret message [25], [26].

Before using color image to create the updated table, we have to sure that the image contains all the values between 0 and 255 , this can be done by replacing 256 pixels in the image with these value, the replacement will not affect the image much, as shown in figures 1 and 2 [27], [28].

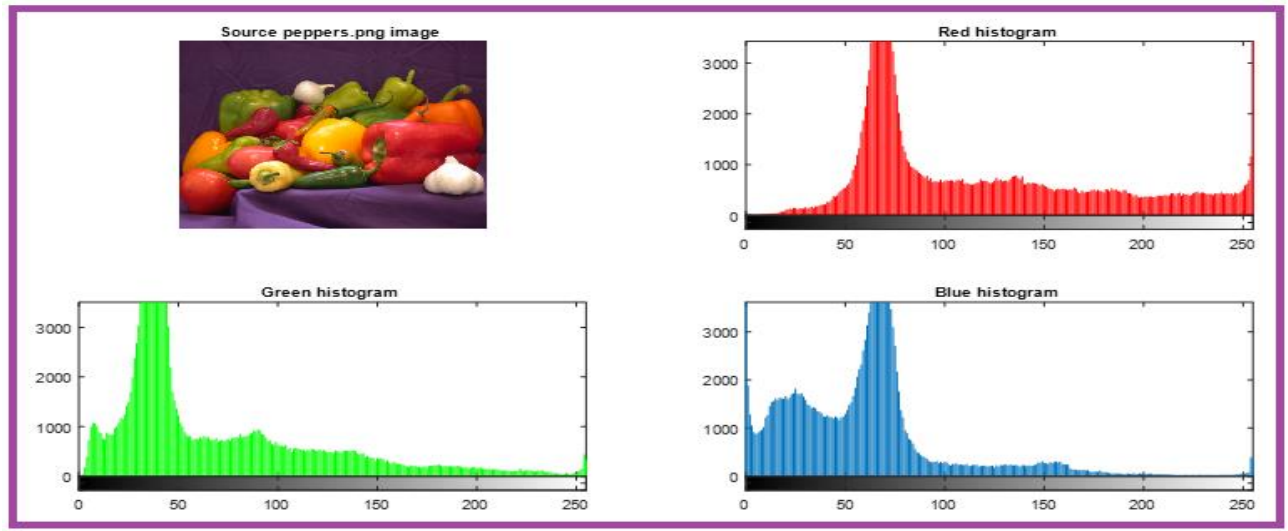

Figure 1: Sample of the color image 


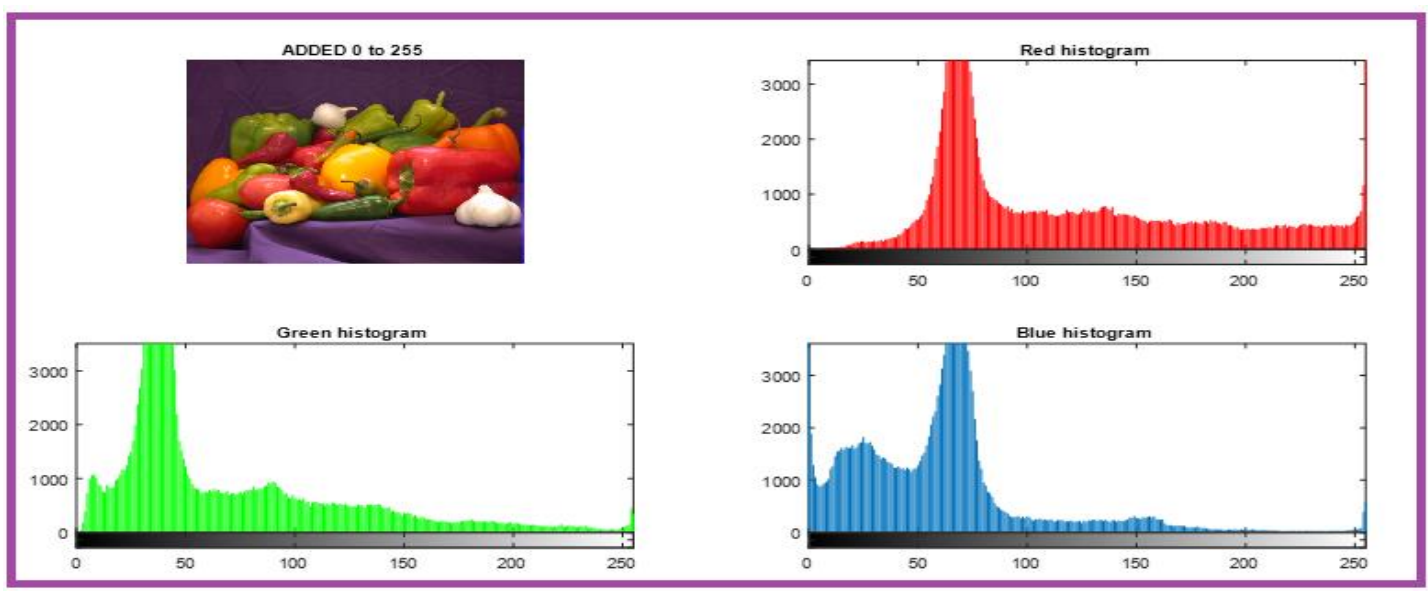

Figure 2: Image after replacing the values of 256 pixels

\section{II- THE PROPOSED METHOD}

The high security level and secret message [29], [32] protection of proposed method can be achieved by selecting a color image to be used to generate the updated table as shown in figure 3 . The selected message is an agreed image between the sender and receiver and it will be available to both of them and there is no need to send it, also the selected source image can be changed whenever the sender and receiver decide to do this.

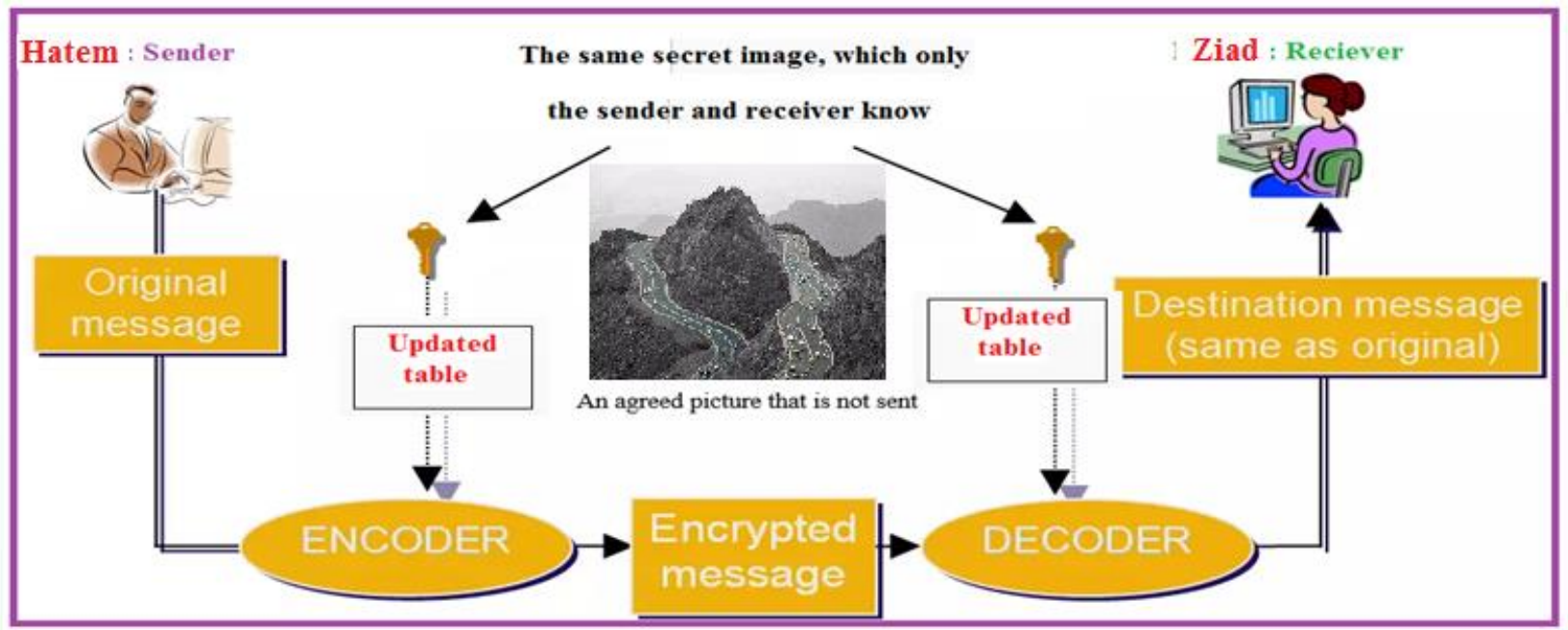

Figure 3: Proposed method diagram

The proposed method can be implemented applying the following steps:

\section{Encryption phase:}

1) Get the source image.

2) Get the image size.

3) Reshape the image into one row matrix.

4) Check whether the row matrix contains all the values between 0 and 255 , if not replace some pixels in the image with the required missing values.

5) For each value in the ASCII table find the first location in the image that contains the ASCII value and store it in the updated table.

6) Get the secret message, and get the message length.

7) For each character in the massage find the associated location in the updated table, and add it to the encrypted message.

8) Send the encrypted message. 


\section{International Journal of Advanced Research in Computer and Communication Engineering}

Vol. 10, Issue 3, March 2021

DOI 10.17148/IJARCCE.2021.10314

\section{Decryption phase}

This phase can be implemented applying the following steps:

1) Get the source image.

2) Get the image size.

3) Reshape the image into one row matrix.

4) Check whether the row matrix contains all the values between 0 and 255 , if not replace some pixels in the image with the required missing values.

5) For each value in the ASCII table find the first location in the image that contains the ASCII value and store it in the updated table.

6) Get the encrypted message, and get the message length.

7) For each value in the massage find the index associated with the location to get the ASCII code, add this code to the decrypted message.

\section{III- IMPLEMENTATION AND EXPERIMENTAL RESULTS}

The proposed method was implemented using matlab; figure 4 shows the ASCII table, while table 1 shows the obtained updated table using the peppers.png image as a source image:

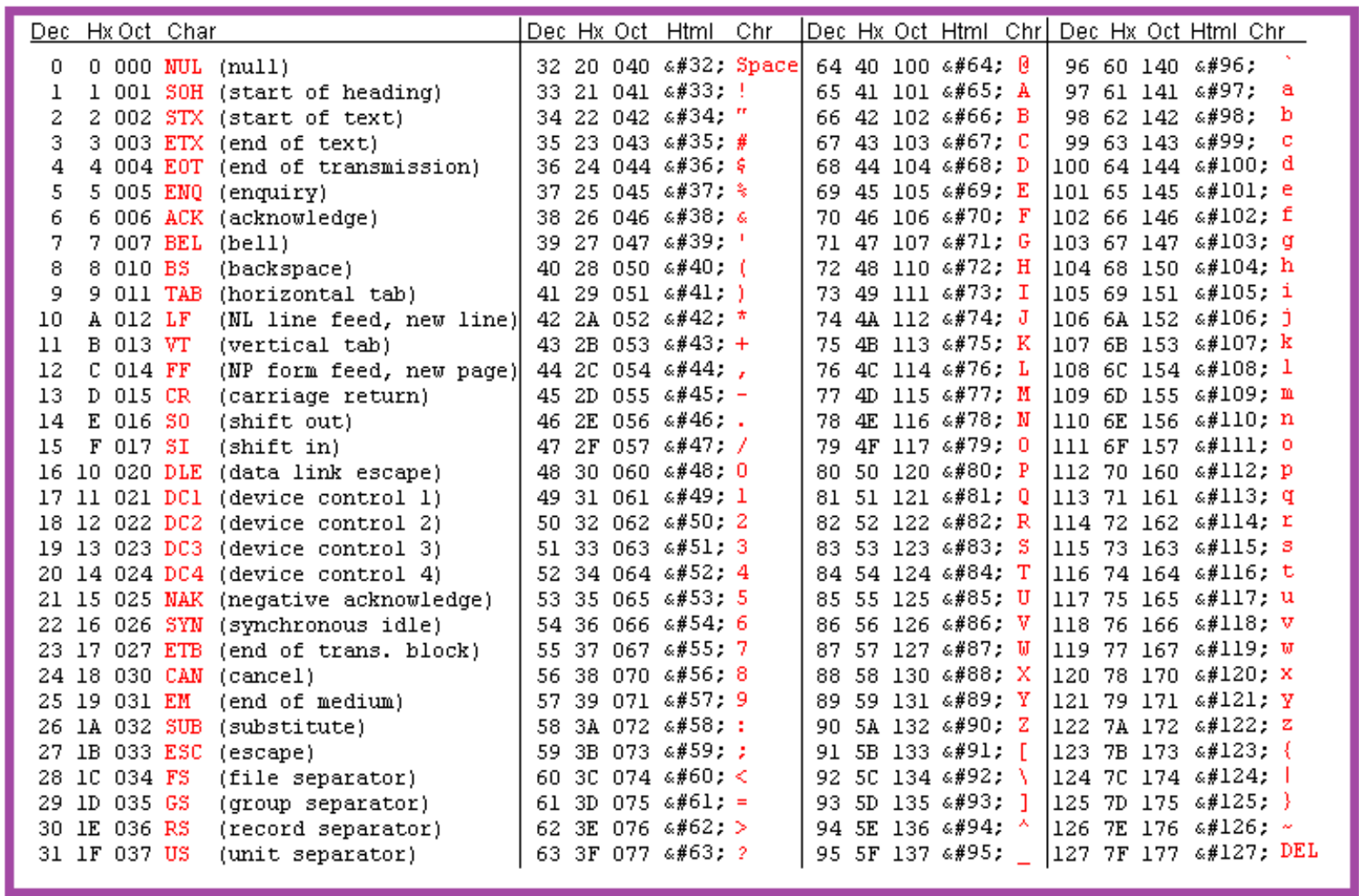

Figure 4: ASCII table 
Vol. 10, Issue 3, March 2021

\section{DOI 10.17148/IJARCCE.2021.10314}

Table 1: Updated table

\begin{tabular}{|c|c|c|c|c|c|c|c|c|c|c|c|c|c|c|c|c|c|}
\hline 0 & $\begin{array}{l}39731 \\
4 \\
\end{array}$ & $\begin{array}{l}3 \\
1 \\
\end{array}$ & 9955 & $\begin{array}{l}6 \\
2 \\
\end{array}$ & 10 & 93 & 693 & $\begin{array}{l}12 \\
4 \\
\end{array}$ & 367 & $\begin{array}{l}15 \\
5\end{array}$ & $\begin{array}{l}258 \\
6 \\
\end{array}$ & $\begin{array}{l}18 \\
6 \\
\end{array}$ & $\begin{array}{l}297 \\
8 \\
\end{array}$ & $\begin{array}{l}21 \\
7 \\
\end{array}$ & $\begin{array}{l}490 \\
1 \\
\end{array}$ & $\begin{array}{l}24 \\
8 \\
\end{array}$ & 8724 \\
\hline 1 & $\begin{array}{l}21309 \\
1\end{array}$ & $\begin{array}{l}3 \\
2\end{array}$ & $\begin{array}{l}2263 \\
0\end{array}$ & $\begin{array}{l}6 \\
3\end{array}$ & 1(A) & 94 & 1469 & $\begin{array}{l}12 \\
5\end{array}$ & 375 & $\begin{array}{l}15 \\
6\end{array}$ & $\begin{array}{l}144 \\
4\end{array}$ & $\begin{array}{l}18 \\
7\end{array}$ & $\begin{array}{l}297 \\
7\end{array}$ & $\begin{array}{l}21 \\
8\end{array}$ & $\begin{array}{l}490 \\
0\end{array}$ & $\begin{array}{l}24 \\
9\end{array}$ & 9107 \\
\hline 2 & $\begin{array}{l}21155 \\
5\end{array}$ & $\begin{array}{l}3 \\
3\end{array}$ & 8802 & $\begin{array}{l}6 \\
4\end{array}$ & $2(B)$ & 95 & 692 & $\begin{array}{l}12 \\
6\end{array}$ & 368 & $\begin{array}{l}15 \\
7\end{array}$ & $\begin{array}{l}145 \\
2\end{array}$ & $\begin{array}{l}18 \\
8\end{array}$ & $\begin{array}{l}259 \\
2\end{array}$ & $\begin{array}{l}21 \\
9\end{array}$ & $\begin{array}{l}527 \\
2\end{array}$ & $\begin{array}{l}25 \\
0\end{array}$ & $\begin{array}{r}1063 \\
9\end{array}$ \\
\hline 3 & $\begin{array}{l}20655 \\
9\end{array}$ & $\begin{array}{l}3 \\
4\end{array}$ & $\begin{array}{l}2032 \\
3\end{array}$ & $\begin{array}{l}6 \\
5\end{array}$ & )$^{16(C}$ & 96 & 357 & $\begin{array}{l}12 \\
7\end{array}$ & 372 & $\begin{array}{l}15 \\
8\end{array}$ & $\begin{array}{l}222 \\
2\end{array}$ & $\begin{array}{l}18 \\
9\end{array}$ & $\begin{array}{l}258 \\
8\end{array}$ & $\begin{array}{l}22 \\
0\end{array}$ & $\begin{array}{l}527 \\
4\end{array}$ & $\begin{array}{l}25 \\
1\end{array}$ & $\begin{array}{r}1102 \\
4\end{array}$ \\
\hline 4 & $\begin{array}{l}20617 \\
6\end{array}$ & $\begin{array}{l}3 \\
5\end{array}$ & 8803 & $\begin{array}{l}6 \\
6\end{array}$ & 3(D) & 97 & 680(a) & $\begin{array}{l}12 \\
8\end{array}$ & 373 & $\begin{array}{l}15 \\
9\end{array}$ & $\begin{array}{l}183 \\
6\end{array}$ & $\begin{array}{l}19 \\
0\end{array}$ & $\begin{array}{l}259 \\
1\end{array}$ & $\begin{array}{l}22 \\
1\end{array}$ & $\begin{array}{l}528 \\
0 \\
\end{array}$ & $\begin{array}{l}25 \\
2\end{array}$ & $\begin{array}{r}1141 \\
4 \\
\end{array}$ \\
\hline 5 & 32224 & $\begin{array}{l}3 \\
6\end{array}$ & 1807 & $\begin{array}{l}6 \\
7\end{array}$ & $31(\mathrm{E})$ & 98 & )$^{1082(b}$ & $\begin{array}{l}12 \\
9\end{array}$ & 370 & $\begin{array}{l}16 \\
0\end{array}$ & $\begin{array}{l}182 \\
4\end{array}$ & $\begin{array}{l}19 \\
1\end{array}$ & $\begin{array}{l}336 \\
3 \\
\end{array}$ & $\begin{array}{l}22 \\
2\end{array}$ & $\begin{array}{l}527 \\
7 \\
\end{array}$ & $\begin{array}{l}25 \\
3\end{array}$ & $\begin{array}{r}1141 \\
7 \\
\end{array}$ \\
\hline 6 & 32223 & $\begin{array}{l}3 \\
7\end{array}$ & 2960 & $\begin{array}{l}6 \\
8\end{array}$ & 24 & 99 & $1125(c)$ & $\begin{array}{l}13 \\
0\end{array}$ & $\begin{array}{l}146 \\
2\end{array}$ & $\begin{array}{l}16 \\
1\end{array}$ & $\begin{array}{l}144 \\
5\end{array}$ & $\begin{array}{l}19 \\
2\end{array}$ & $\begin{array}{l}259 \\
0\end{array}$ & $\begin{array}{l}22 \\
3\end{array}$ & $\begin{array}{l}527 \\
5\end{array}$ & $\begin{array}{l}25 \\
4\end{array}$ & $\begin{array}{r}1179 \\
8\end{array}$ \\
\hline 7 & 26852 & $\begin{array}{l}3 \\
8\end{array}$ & 1422 & $\begin{array}{l}6 \\
9\end{array}$ & 30 & $\begin{array}{l}10 \\
0\end{array}$ & )$^{1081(d}$ & $\begin{array}{l}13 \\
1\end{array}$ & 371 & $\begin{array}{l}16 \\
2\end{array}$ & $\begin{array}{l}144 \\
6\end{array}$ & $\begin{array}{l}19 \\
3\end{array}$ & $\begin{array}{l}258 \\
9 \\
\end{array}$ & $\begin{array}{l}22 \\
4\end{array}$ & $\begin{array}{l}527 \\
6 \\
\end{array}$ & $\begin{array}{l}25 \\
5\end{array}$ & $\begin{array}{r}1218 \\
1 \\
\end{array}$ \\
\hline 8 & 24549 & $\begin{array}{l}3 \\
9\end{array}$ & 1037 & $\begin{array}{l}7 \\
0\end{array}$ & 25 & $\begin{array}{l}10 \\
1\end{array}$ & 691(e) & $\begin{array}{l}13 \\
2\end{array}$ & 378 & $\begin{array}{l}16 \\
3\end{array}$ & $\begin{array}{l}144 \\
9\end{array}$ & $\begin{array}{l}19 \\
4\end{array}$ & $\begin{array}{l}374 \\
5\end{array}$ & $\begin{array}{l}22 \\
5\end{array}$ & $\begin{array}{l}565 \\
6\end{array}$ & & \\
\hline 9 & 27235 & $\begin{array}{l}4 \\
0\end{array}$ & 270 & $\begin{array}{l}7 \\
1\end{array}$ & 57 & $\begin{array}{l}10 \\
2\end{array}$ & 681(f) & $\begin{array}{l}13 \\
3\end{array}$ & $\begin{array}{l}184 \\
7\end{array}$ & $\begin{array}{l}16 \\
4\end{array}$ & $\begin{array}{l}145 \\
0\end{array}$ & $\begin{array}{l}19 \\
5\end{array}$ & $\begin{array}{l}335 \\
8\end{array}$ & $\begin{array}{l}22 \\
6\end{array}$ & $\begin{array}{l}565 \\
8\end{array}$ & & \\
\hline $\begin{array}{l}1 \\
0\end{array}$ & 24165 & $\begin{array}{l}4 \\
1\end{array}$ & 272 & $\begin{array}{l}7 \\
2\end{array}$ & 116 & $\begin{array}{l}10 \\
3\end{array}$ & 1080 & $\begin{array}{l}13 \\
4\end{array}$ & $\begin{array}{l}107 \\
3\end{array}$ & $\begin{array}{l}16 \\
5\end{array}$ & $\begin{array}{l}144 \\
7\end{array}$ & $\begin{array}{l}19 \\
6\end{array}$ & $\begin{array}{l}297 \\
4\end{array}$ & $\begin{array}{l}22 \\
7\end{array}$ & $\begin{array}{l}565 \\
9\end{array}$ & & \\
\hline $\begin{array}{l}1 \\
1\end{array}$ & 24933 & $\begin{array}{l}4 \\
2\end{array}$ & 653 & $\begin{array}{l}7 \\
3\end{array}$ & 93 & $\begin{array}{l}10 \\
4\end{array}$ & 1127 & $\begin{array}{l}13 \\
5\end{array}$ & $\begin{array}{l}106 \\
3\end{array}$ & $\begin{array}{l}16 \\
6\end{array}$ & $\begin{array}{l}183 \\
3\end{array}$ & $\begin{array}{l}19 \\
7\end{array}$ & $\begin{array}{l}297 \\
0 \\
\end{array}$ & $\begin{array}{l}22 \\
8\end{array}$ & $\begin{array}{l}603 \\
8 \\
\end{array}$ & & \\
\hline $\begin{array}{l}1 \\
2\end{array}$ & 26084 & $\begin{array}{l}4 \\
3\end{array}$ & 247 & $\begin{array}{l}7 \\
4\end{array}$ & 115 & $\begin{array}{l}10 \\
5\end{array}$ & 1060 & $\begin{array}{l}13 \\
6\end{array}$ & $\begin{array}{l}223 \\
1\end{array}$ & $\begin{array}{l}16 \\
7\end{array}$ & $\begin{array}{l}409 \\
9\end{array}$ & $\begin{array}{l}19 \\
8\end{array}$ & $\begin{array}{l}297 \\
3\end{array}$ & $\begin{array}{l}22 \\
9\end{array}$ & $\begin{array}{l}604 \\
0\end{array}$ & & \\
\hline $\begin{array}{l}1 \\
3\end{array}$ & 28002 & $\begin{array}{l}4 \\
4\end{array}$ & 268 & $\begin{array}{l}7 \\
5\end{array}$ & 117 & $\begin{array}{l}10 \\
6\end{array}$ & 358 & $\begin{array}{l}13 \\
7\end{array}$ & $\begin{array}{l}107 \\
2\end{array}$ & $\begin{array}{l}16 \\
8\end{array}$ & $\begin{array}{l}182 \\
5\end{array}$ & $\begin{array}{l}19 \\
9\end{array}$ & $\begin{array}{l}297 \\
1\end{array}$ & $\begin{array}{l}23 \\
0\end{array}$ & $\begin{array}{l}566 \\
4\end{array}$ & & \\
\hline $\begin{array}{l}1 \\
4\end{array}$ & 23013 & $\begin{array}{l}4 \\
5\end{array}$ & 263 & $\begin{array}{l}7 \\
6\end{array}$ & 107 & $\begin{array}{l}10 \\
7\end{array}$ & 359 & $\begin{array}{l}13 \\
8\end{array}$ & $\begin{array}{l}223 \\
0\end{array}$ & $\begin{array}{l}16 \\
9\end{array}$ & $\begin{array}{l}221 \\
7\end{array}$ & $\begin{array}{l}20 \\
0\end{array}$ & $\begin{array}{l}373 \\
9\end{array}$ & $\begin{array}{l}23 \\
1\end{array}$ & $\begin{array}{l}604 \\
2\end{array}$ & & \\
\hline $\begin{array}{l}1 \\
5\end{array}$ & 23397 & $\begin{array}{l}4 \\
6\end{array}$ & 248 & $\begin{array}{l}7 \\
7\end{array}$ & 133 & $\begin{array}{l}10 \\
8\end{array}$ & 1130 & $\begin{array}{l}13 \\
9\end{array}$ & $\begin{array}{l}146 \\
0\end{array}$ & $\begin{array}{l}17 \\
0\end{array}$ & $\begin{array}{l}260 \\
2\end{array}$ & $\begin{array}{l}20 \\
1\end{array}$ & $\begin{array}{l}335 \\
2\end{array}$ & $\begin{array}{l}23 \\
2\end{array}$ & $\begin{array}{l}604 \\
5\end{array}$ & & \\
\hline $\begin{array}{l}1 \\
6\end{array}$ & 24932 & $\begin{array}{l}4 \\
7\end{array}$ & 260 & $\begin{array}{l}7 \\
8\end{array}$ & 242 & $\begin{array}{l}10 \\
9\end{array}$ & 360 & $\begin{array}{l}14 \\
0\end{array}$ & $\begin{array}{l}106 \\
4\end{array}$ & $\begin{array}{l}17 \\
1\end{array}$ & $\begin{array}{l}182 \\
6\end{array}$ & $\begin{array}{l}20 \\
2\end{array}$ & $\begin{array}{l}335 \\
4\end{array}$ & $\begin{array}{l}23 \\
3\end{array}$ & $\begin{array}{l}604 \\
6\end{array}$ & & \\
\hline $\begin{array}{l}1 \\
7\end{array}$ & 22629 & $\begin{array}{l}4 \\
8\end{array}$ & 644 & $\begin{array}{l}7 \\
9\end{array}$ & 294 & $\begin{array}{l}11 \\
0\end{array}$ & 361 & $\begin{array}{l}14 \\
1\end{array}$ & $\begin{array}{l}106 \\
5\end{array}$ & $\begin{array}{l}17 \\
2\end{array}$ & $\begin{array}{l}182 \\
9\end{array}$ & $\begin{array}{l}20 \\
3\end{array}$ & $\begin{array}{l}412 \\
4\end{array}$ & $\begin{array}{l}23 \\
4\end{array}$ & $\begin{array}{l}642 \\
2\end{array}$ & & \\
\hline $\begin{array}{l}1 \\
8\end{array}$ & 24164 & $\begin{array}{l}4 \\
9\end{array}$ & 249 & $\begin{array}{l}8 \\
0\end{array}$ & 295 & $\begin{array}{l}11 \\
1\end{array}$ & 684 & $\begin{array}{l}14 \\
2\end{array}$ & $\begin{array}{l}107 \\
1\end{array}$ & $\begin{array}{l}17 \\
3\end{array}$ & $\begin{array}{l}182 \\
7\end{array}$ & $\begin{array}{l}20 \\
4\end{array}$ & $\begin{array}{l}335 \\
3\end{array}$ & $\begin{array}{l}23 \\
5\end{array}$ & $\begin{array}{l}642 \\
8\end{array}$ & & \\
\hline $\begin{array}{l}1 \\
9\end{array}$ & 22245 & $\begin{array}{l}5 \\
0\end{array}$ & 630 & $\begin{array}{l}8 \\
1\end{array}$ & 108 & $\begin{array}{l}11 \\
2\end{array}$ & 384 & $\begin{array}{l}14 \\
3\end{array}$ & $\begin{array}{l}107 \\
0\end{array}$ & $\begin{array}{l}17 \\
4\end{array}$ & $\begin{array}{l}183 \\
1\end{array}$ & $\begin{array}{l}20 \\
5\end{array}$ & $\begin{array}{l}451 \\
5\end{array}$ & $\begin{array}{l}23 \\
6\end{array}$ & $\begin{array}{l}643 \\
0\end{array}$ & & \\
\hline $\begin{array}{l}2 \\
0\end{array}$ & 21861 & $\begin{array}{l}5 \\
1\end{array}$ & 257 & $\begin{array}{l}8 \\
2\end{array}$ & 297 & $\begin{array}{l}11 \\
3\end{array}$ & 1078 & $\begin{array}{l}14 \\
4\end{array}$ & $\begin{array}{l}106 \\
6\end{array}$ & $\begin{array}{l}17 \\
5\end{array}$ & $\begin{array}{l}182 \\
8\end{array}$ & $\begin{array}{l}20 \\
6\end{array}$ & $\begin{array}{l}373 \\
4 \\
\end{array}$ & $\begin{array}{l}23 \\
7\end{array}$ & $\begin{array}{l}681 \\
1 \\
\end{array}$ & & \\
\hline
\end{tabular}


International Journal of Advanced Research in Computer and Communication Engineering

Vol. 10, Issue 3, March 2021

DOI 10.17148/IJARCCE.2021.10314

\begin{tabular}{|c|c|c|c|c|c|c|c|c|c|c|c|c|c|c|c|}
\hline $\begin{array}{l}2 \\
1\end{array}$ & 24550 & $\begin{array}{l}5 \\
2\end{array}$ & 250 & $\begin{array}{l}8 \\
3\end{array}$ & 110 & $\begin{array}{l}11 \\
4\end{array}$ & 383 & $\begin{array}{l}14 \\
5\end{array}$ & $\begin{array}{l}106 \\
7\end{array}$ & $\begin{array}{l}17 \\
6\end{array}$ & $\begin{array}{l}221 \\
4\end{array}$ & $\begin{array}{l}20 \\
7\end{array}$ & $\begin{array}{l}450 \\
5\end{array}$ & $\begin{array}{l}23 \\
8\end{array}$ & $\begin{array}{l}680 \\
6\end{array}$ \\
\hline $\begin{array}{l}2 \\
2\end{array}$ & 21477 & $\begin{array}{l}5 \\
3\end{array}$ & 256 & $\begin{array}{l}8 \\
4\end{array}$ & 298 & $\begin{array}{l}11 \\
5\end{array}$ & 768 & $\begin{array}{l}14 \\
6\end{array}$ & $\begin{array}{l}106 \\
8\end{array}$ & $\begin{array}{l}17 \\
7\end{array}$ & $\begin{array}{l}221 \\
3\end{array}$ & $\begin{array}{l}20 \\
8\end{array}$ & $\begin{array}{l}373 \\
5\end{array}$ & $\begin{array}{l}23 \\
9\end{array}$ & $\begin{array}{l}680 \\
5\end{array}$ \\
\hline $\begin{array}{l}2 \\
3\end{array}$ & 21093 & $\begin{array}{l}5 \\
4\end{array}$ & 634 & $\begin{array}{l}8 \\
5\end{array}$ & 305 & $\begin{array}{l}11 \\
6\end{array}$ & 747 & $\begin{array}{l}14 \\
7\end{array}$ & $\begin{array}{l}338 \\
3\end{array}$ & $\begin{array}{l}17 \\
8\end{array}$ & $\begin{array}{l}258 \\
7\end{array}$ & $\begin{array}{l}20 \\
9\end{array}$ & $\begin{array}{l}411 \\
8\end{array}$ & $\begin{array}{l}24 \\
0\end{array}$ & $\begin{array}{l}680 \\
8\end{array}$ \\
\hline $\begin{array}{l}2 \\
4\end{array}$ & 20709 & $\begin{array}{l}5 \\
5\end{array}$ & 638 & $\begin{array}{l}8 \\
6\end{array}$ & 302 & $\begin{array}{l}11 \\
7\end{array}$ & 750 & $\begin{array}{l}14 \\
8\end{array}$ & $\begin{array}{l}184 \\
2\end{array}$ & $\begin{array}{l}17 \\
9\end{array}$ & $\begin{array}{l}259 \\
9\end{array}$ & $\begin{array}{l}21 \\
0\end{array}$ & $\begin{array}{l}411 \\
9\end{array}$ & $\begin{array}{l}24 \\
1\end{array}$ & $\begin{array}{l}719 \\
0\end{array}$ \\
\hline $\begin{array}{l}2 \\
5\end{array}$ & 20325 & $\begin{array}{l}5 \\
6\end{array}$ & 220 & $\begin{array}{l}8 \\
7\end{array}$ & 303 & $\begin{array}{l}11 \\
8\end{array}$ & 362 & $\begin{array}{l}14 \\
9\end{array}$ & $\begin{array}{l}182 \\
3\end{array}$ & $\begin{array}{l}18 \\
0\end{array}$ & $\begin{array}{l}220 \\
6\end{array}$ & $\begin{array}{l}21 \\
1\end{array}$ & $\begin{array}{l}450 \\
2\end{array}$ & $\begin{array}{l}24 \\
2\end{array}$ & $\begin{array}{l}757 \\
6\end{array}$ \\
\hline $\begin{array}{l}2 \\
6\end{array}$ & 9186 & $\begin{array}{l}5 \\
7\end{array}$ & 14 & $\begin{array}{l}8 \\
8\end{array}$ & 304 & $\begin{array}{l}11 \\
9\end{array}$ & 766 & $\begin{array}{l}15 \\
0\end{array}$ & $\begin{array}{l}144 \\
3\end{array}$ & $\begin{array}{l}18 \\
1\end{array}$ & $\begin{array}{l}375 \\
9\end{array}$ & $\begin{array}{l}21 \\
2\end{array}$ & $\begin{array}{l}450 \\
0\end{array}$ & $\begin{array}{l}24 \\
3\end{array}$ & $\begin{array}{l}757 \\
3\end{array}$ \\
\hline $\begin{array}{l}2 \\
7\end{array}$ & 21476 & $\begin{array}{l}5 \\
8\end{array}$ & 11 & $\begin{array}{l}8 \\
9\end{array}$ & 355 & $\begin{array}{l}12 \\
0\end{array}$ & 365 & $\begin{array}{l}15 \\
1\end{array}$ & $\begin{array}{l}184 \\
0\end{array}$ & $\begin{array}{l}18 \\
2\end{array}$ & $\begin{array}{l}221 \\
0\end{array}$ & $\begin{array}{l}21 \\
3\end{array}$ & $\begin{array}{l}450 \\
1\end{array}$ & $\begin{array}{l}24 \\
4\end{array}$ & $\begin{array}{l}757 \\
4\end{array}$ \\
\hline $\begin{array}{l}2 \\
8\end{array}$ & 20324 & $\begin{array}{l}5 \\
9\end{array}$ & 17 & $\begin{array}{l}9 \\
0\end{array}$ & 1105 & $\begin{array}{l}12 \\
1\end{array}$ & 363 & $\begin{array}{l}15 \\
2\end{array}$ & $\begin{array}{l}222 \\
4\end{array}$ & $\begin{array}{l}18 \\
3\end{array}$ & $\begin{array}{l}259 \\
5\end{array}$ & $\begin{array}{l}21 \\
4\end{array}$ & $\begin{array}{l}488 \\
4\end{array}$ & $\begin{array}{l}24 \\
5\end{array}$ & $\begin{array}{l}834 \\
2\end{array}$ \\
\hline $\begin{array}{l}2 \\
9\end{array}$ & 9571 & $\begin{array}{l}6 \\
0\end{array}$ & 27 & $\begin{array}{l}9 \\
1\end{array}$ & 333 & $\begin{array}{l}12 \\
2\end{array}$ & 1076 & $\begin{array}{l}15 \\
3\end{array}$ & $\begin{array}{l}415 \\
4\end{array}$ & $\begin{array}{l}18 \\
4\end{array}$ & $\begin{array}{l}220 \\
7\end{array}$ & $\begin{array}{l}21 \\
5\end{array}$ & $\begin{array}{l}565 \\
0\end{array}$ & $\begin{array}{l}24 \\
6\end{array}$ & $\begin{array}{l}834 \\
1\end{array}$ \\
\hline $\begin{array}{l}3 \\
0\end{array}$ & 9570 & $\begin{array}{l}6 \\
1\end{array}$ & 9955 & $\begin{array}{l}9 \\
2\end{array}$ & 356 & $\begin{array}{l}12 \\
3\end{array}$ & 366 & $\begin{array}{l}15 \\
4\end{array}$ & $\begin{array}{l}145 \\
5\end{array}$ & $\begin{array}{l}18 \\
5\end{array}$ & $\begin{array}{l}220 \\
8\end{array}$ & $\begin{array}{l}21 \\
6\end{array}$ & $\begin{array}{l}489 \\
1\end{array}$ & $\begin{array}{l}24 \\
7\end{array}$ & $\begin{array}{l}834 \\
0\end{array}$ \\
\hline
\end{tabular}

As we said, the source image can be changed, and here the encrypted versions for the same secret message will be different as shown in figure 5:

\begin{tabular}{|c|c|c|c|c|c|}
\hline $\mathbf{O M}$ & EM1 & EM2 & EM3 & EM4 & \multirow{20}{*}{$\begin{array}{c}\text { EMi:Encrypted Message } \\
\text { using image } i\end{array}$} \\
\hline 90 & 29 & 668 & 55 & 222 & \\
\hline 105 & 61 & 1348 & 243 & 260 & \\
\hline 97 & 76 & 1009 & 222 & 311 & \\
\hline 100 & 33 & 702 & 224 & 270 & \\
\hline 32 & 410 & 1656 & 24 & 9946 & \\
\hline 65 & 99 & 2109 & 46 & 68 & \\
\hline 108 & 30 & 908 & 115 & 214 & \\
\hline 113 & 51 & 2115 & 494 & 316 & \\
\hline 97 & 76 & 1009 & 222 & 311 & \\
\hline 100 & 33 & 702 & 224 & 270 & \\
\hline 105 & 61 & 1348 & 243 & 260 & \\
\hline 32 & 410 & 1656 & 24 & 9946 & \\
\hline 66 & 96 & 2686 & 59 & 22 & \\
\hline 65 & 99 & 2109 & 46 & 68 & \\
\hline 85 & 85 & 1212 & 539 & 18 & \\
\hline 32 & 410 & 1656 & 24 & 9946 & \\
\hline 70 & 244 & 852 & 57 & 2 & \\
\hline 69 & 246 & 2108 & 58 & 77 & \\
\hline 84 & 388 & 401 & 855 & 17 & \\
\hline
\end{tabular}

Figure 5: Different encrypted messages using different images

Here we have to notice that using any color image as a source one we can encrypt-decrypt messages with variable length, and the message length can be unlimited. The following image shown in figure 6 was taken as a source image, various messages were encrypted-decrypted using this image, the encryption and decryption times were measured, table 2 shows the obtained experimental results: 


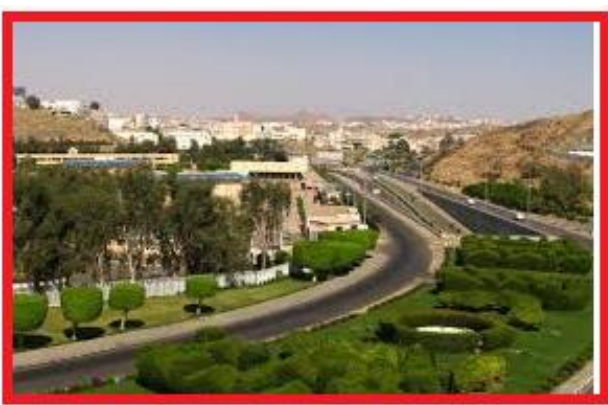

Figure 6: Source image (image size $=150849$ bytes)

Table 2: Obtained results

\begin{tabular}{|c|c|c|c|}
\hline Message length & Encryption time(Second) & decryption time (Seconds) & Throughput \\
\hline 19 & 0.270000 & 0.270000 & 70 \\
\hline 32 & 0.274000 & 0.274000 & 120 \\
\hline 52 & 0.281000 & 0.281000 & 350 \\
\hline 104 & 0.296000 & 0.296000 & 690 \\
\hline 208 & 0.302000 & 0.302000 & 1350 \\
\hline 416 & 0.309000 & 0.309000 & 45000 \\
\hline 1664 & 0.370000 & 0.370000 & 17290 \\
\hline 6656 & 0.385000 & 0.385000 & 103390 \\
\hline 53248 & 0.515000 & 0.515000 & 116140 \\
\hline
\end{tabular}

From the obtained results shown in table 2 we can raise the following facts:

The secret message length can be bigger than the image size.

We can use any color image as a source image to encrypt-decrypt the secret message.

The proposed method is very flexible by changing the source image whenever we want.

There is no need to send the source image, it must be kept in secret by both the sender and receiver, and thus we can achieve a high level of security.

- $\quad$ The proposed method is very efficient by providing a small encryption-decryption time, thus the providing method maximizes the method throughput (number of encrypted/decrypted bytes per second). See figures 7 and 8 .

The proposed method is very simple to be implemented

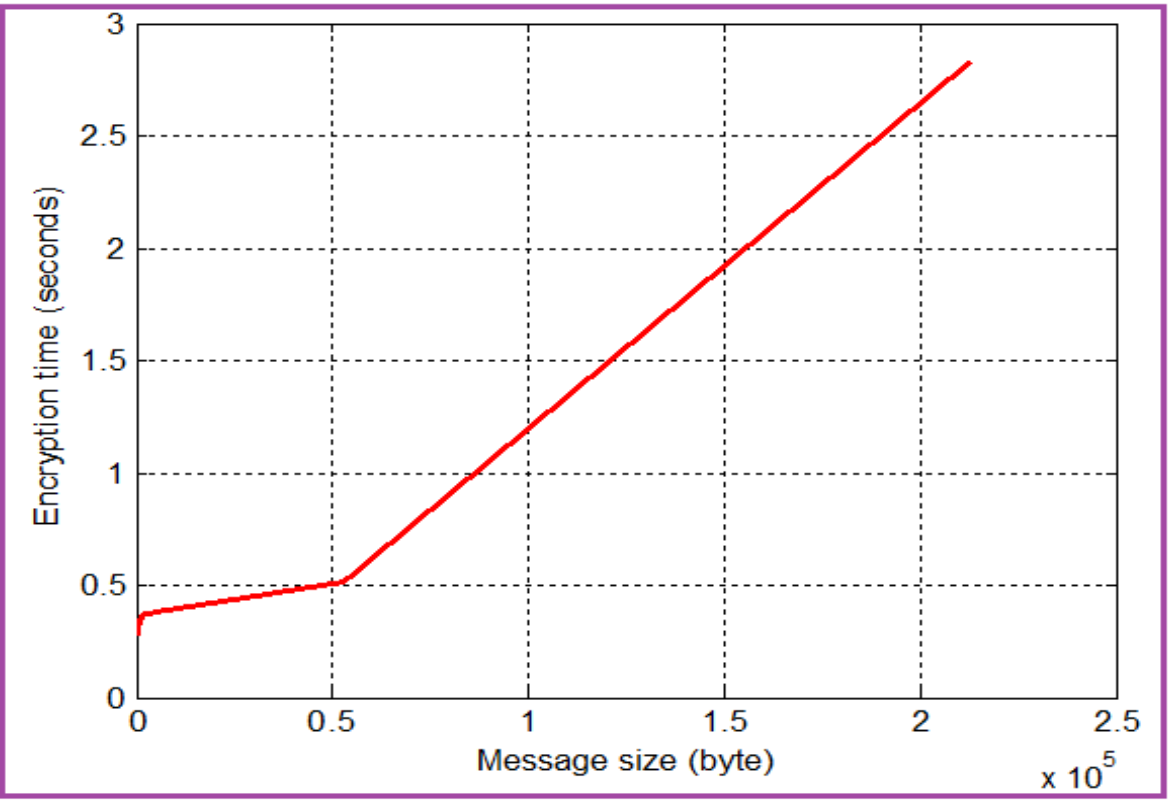

Figure 7: Relationship between message length and encryption time 


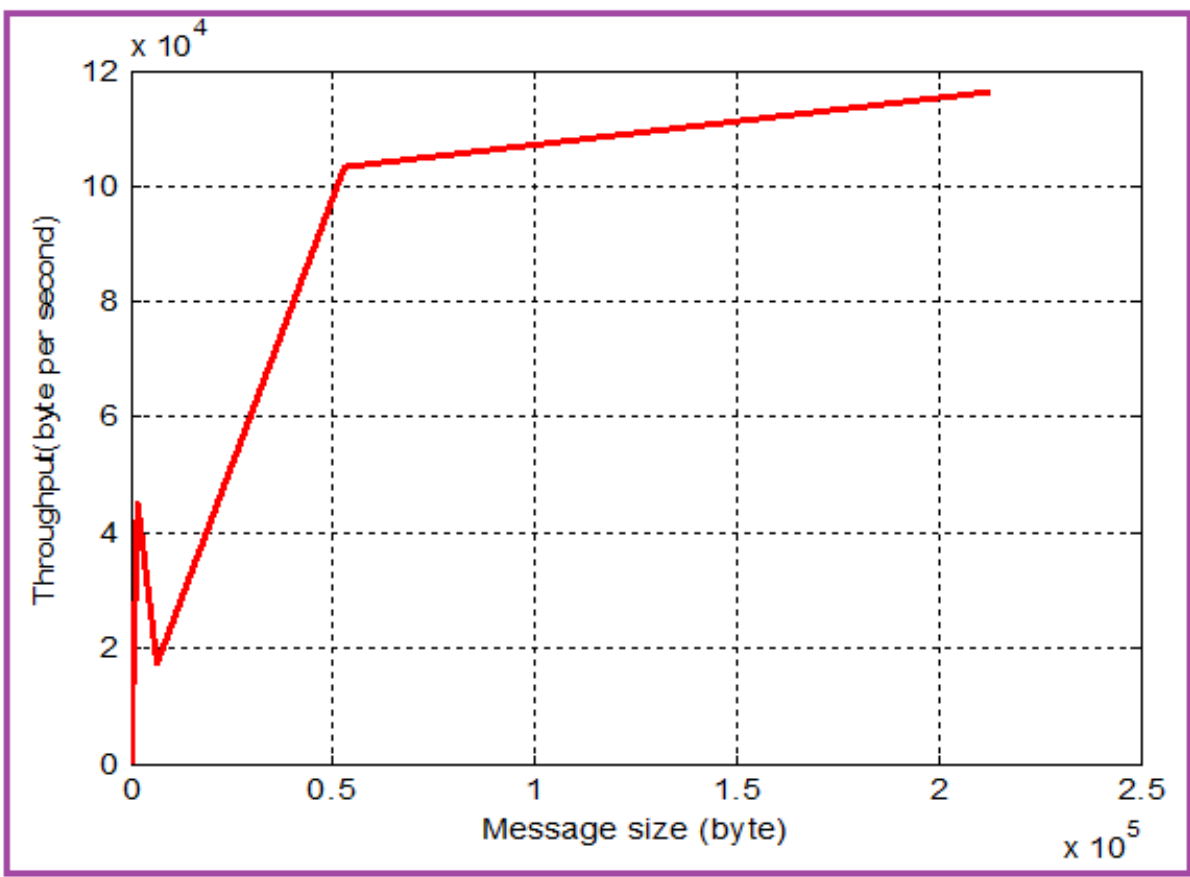

Figure 8: Relationship between message length and method throughput

For messages with KB sizes, the throughput was compared with DES and AES results [33] and the proposed method gave better results by raising the throughput from 4.4 to $116 \mathrm{~Kb}$ per second.

\section{IV-Conclusion}

A method of secret message encryption-decryption was proposed, tested and implemented. This method is based on replacing the ASCII table by a new updated table which contains the first occurrence (location) of the ASCII symbol in the source image.

The proposed method has a high level of security, this can be achieved by keeping the source image unknown (secret) to any third party, and by selecting an image with huge size, also there is a possibility to change the source image from time to time.

The experimental results showed that the proposed method is very efficient by maximizing the method throughput

\section{REFERENCES}

[1] Amjad Y Hindi, Majed O Dwairi, Ziad A AlQadi, A Novel Technique for Data Steganography, Engineering, Technology \& Applied Science Research, vol. 9, issue 6, pp. 4942-4945, 2019.

[2] Ziad AlQadi, M Elsayyed Hussein, Window Averaging Method to Create a Feature Victor for RGB Color Image, International Journal of Computer Science and Mobile Computing, vol. 6, issue 2, pp. 60-66, 2017.

[3] Ziad AlQadi, analysis of stream cipher security algorithm, Journal of Information and Computing Science, vol. 2, issue 4, pp. $288-298,2007$.

[4] Prof. Yousif El Tous Prof. Ziad Alqadi, Dr. Mohammad S. Khrisat, Message segmentation and image blocking to secure data steganography, International Journal of Computer Science and Mobile Computing, vol. 9, issue 8, pp.75-82, 2020.

[5] Belal Zahran Rashad J. Rasras, Ziad Alqadi, Mutaz Rasmi Abu Sara, Developing new multilevel security algorithm for data encryptiondecryption (MLS_ED), International Journal of Advanced Trends in Computer Science and Engineering, vol. 8, issue 6, pp.3228-3235, 2020.

[6] Dr. Majed Omar Dwairi Prof. Ziad Alqadi, Dr. Mohammad S. Khrisat, Dr. Amjad Hindi, Simple and Highly Secure, Efficient and Accurate Method (SSEAM) to Encrypt-Decrypt Color Image, International Journal of Advanced Research in Computer and Communication Engineering, vol. 9, issue 4, pp. 64-69, 2020.

[7] Majed O Al-Dwairi, Ziad A Alqadi, Amjad A Abujazar, Rushdi Abu Zneit, Optimized true-color image processing, World Applied Sciences Journal, vol. 8, issue 10, pp. 1175-1182, 2010.

[8] Jamil Al Azzeh, Hussein Alhatamleh, Ziad A Alqadi, Mohammad Khalil Abuzalata, Creating a Color Map to be used to Convert a Gray Image to Color Image, International Journal of Computer Applications, vol. 153, issue 2, pp. 31-34, 2016.

[9] Qazem Jaber Ziad Alqadi, Jamil azza, Statistical analysis of methods used to enhance color image histogram, XX International scientific and technical conference, 2017.

[10] Bassam Subaih Ziad Alqadi, Hamdan Mazen, A Methodology to Analyze Objects in Digital Image using Matlab, International Journal of Computer Science \& Mobile Computing, vol. 5, issue 11, pp. 21-28, 2016.

[11]Mazen A.Hamdan Bassam M.Subaih, Prof. Ziad A. Alqadi, Extracting Isolated Words from an Image of Text, International Journal of Computer Science \& Mobile Computing, vol. 5, issue 11, pp. 29-36, 2016.

[12] Dr. Amjad Hindi, Dr. Majed Omar Dwairi, Prof. Ziad Alqadi, Analysis of Procedures used to build an Optimal Fingerprint Recognition System, International Journal of Computer Science and Mobile Computing, vol. 9, issue 2, pp. 21 - 37, 2020.

[13]Ziad A AlQadi Amjad Y Hindi, O Dwairi Majed, PROCEDURES FOR SPEECH RECOGNITION USING LPC AND ANN, International Journal of Engineering Technology Research \& Management, vol. 4, issue 2, pp. 48-55, 2020. 


\section{International Journal of Advanced Research in Computer and Communication Engineering}

Vol. 10, Issue 3, March 2021

DOI 10.17148/IJARCCE.2021.10314

[14] Aws AlQaisi, Mokhled AlTarawneh, Ziad A. Alqadi, Ahmad A. Sharadqah, Analysis of Color Image Features Extraction using Texture Methods, TELKOMNIKA, vol. 17, issue 3, pp. 1220-1225, 2019.

[15]Ahmad Sharadqh Naseem Asad, Ismail Shayeb, Qazem Jaber, Belal Ayyoub, Ziad Alqadi, Creating a Stable and Fixed Features Array for Digital Color Image, IJCSMC, vol. 8, issue 8, pp. 50-56, 2019

[16] Ziad Alqadi, Dr. Mohammad S. Khrisat, Dr. Amjad Hindi, Dr. Majed Omar Dwairi, VALUABLE WAVELET PACKET INFORMATION TO ANALYZE COLOR IMAGES FEATURES, International Journal of Current Advanced Research, vol. 9, issue 2, pp. 2319,2020.

[17] Majed O. Al-Dwairi, Amjad Y. Hendi, Mohamed S. Soliman, Ziad A.A. Alqadi, A new method for voice signal features creation, International Journal of Electrical and Computer Engineering (IJECE), vol. 9, issue 5, pp. 4092-4098, 2019.

[18]Ziad AlQadi, M Elsayyed Hussein, Window Averaging Method to Create a Feature Victor for RGB Color Image, International Journal of Computer Science and Mobile Computing, vol. 6, issue 2, pp. 60-66, 2017.

[19] Bilal Zahran Belal Ayyoub, Jihad Nader, Ziad Al-Qadi, Suggested Method to Create Color Image Features Victor, Journal of Engineering and Applied Sciences, vol. 14, issue 1, pp. 2203-2207, 2019.

[20]Majed O. Al-Dwairi, Amjad Y. Hendi, Mohamed S. Soliman, Ziad A.A. Alqadi, A new method for voice signal features creation, International Journal of Electrical and Computer Engineering (IJECE), vol. 9, issue 5, pp. 4092-4098, 2019.

[21]Ayman Al-Rawashdeh, Ziad Al-Qadi, Using wave equation to extract digital signal features, Engineering, Technology \& Applied Science Research, vol. 8, issue 4, pp. 1356-1359, 2018.

[22]Ahmad Sharadqh Naseem Asad, Ismail Shayeb, Qazem Jaber, Belal Ayyoub, Ziad Alqadi, Creating a Stable and Fixed Features Array for Digital Color Image, IJCSMC, vol. 8, issue 8, pp. 50-56, 2019.

[23]ZIAD ALQADI, A MODIFIED LBP METHOD TO EXTRACT FEATURES FROM COLOR IMAGES, Journal of Theoretical and Applied Information Technology, vol. 96, issue 10, pp. 3014-3024,2018.

[24]Aws Al-Qaisi, Saleh A Khawatreh, Ahmad A Sharadqah, Ziad A Alqadi, Wave File Features Extraction Using Reduced LBP, International Journal of Electrical and Computer Engineering, vol. 8, issue 5, pp. 2780-2787, 2018

[25]Jihad Nader Ismail Shayeb, Ziad Alqadi, Jihad Nader, Analysis of digital voice features extraction methods, International Journal of Educational Research and Development, vol. 1, issue 4, pp. 49-55, 2019.

[26]Ahmad Sharadqh Jamil Al-Azzeh , Rashad Rasras , Ziad Alqadi , Belal Ayyoub, Adaptation of matlab K-means clustering function to create Color Image Features, International Journal of Research in Advanced Engineering and Technology, vol. 5, issue 2, pp. 10-18, 2019.

[27] Yousf Eltous Ziad A. AlQadi, Ghazi M. Qaryouti, Mohammad Abuzalata, ANALYSIS OF DIGITAL SIGNAL FEATURES EXTRACTION BASED ON KMEANS CLUSTERING, International Journal of Engineering Technology Research \& Management, vol. 4, issue 1, pp. 66-75, 2020.

[28]Amjad Y Hindi, Majed O Dwairi, Ziad A AlQadi, Creating Human Speech Identifier using WPT, International Journal of Computer Science and Mobile Computing, vol. 9, issue 2, pp. 117-123, 2020.

[29]Amjad Hindi, Majed Omar Dwairi, Ziad Alqadi, Analysis of Digital Signals using Wavelet Packet Tree, IJCSMC, vol. 9, issue 2, pp. 96-103, 2020.

[30] Dr. Amjad Hindi, Dr. Ghazi M. Qaryouti, Prof. Yousif Eltous, Prof. Mohammad Abuzalata, Prof. Ziad Alqadi, Color Image Compression using Linear Prediction Coding, International Journal of Computer Science and Mobile Computing, vol. 9, issue 2, pp. 13 - $20,2020$.

[31] Ziad Alqad, Majid Oraiqat, Hisham Almujafet, Salah Al-Saleh, Hind Al Husban, Soubhi Al-Rimawi,A New Approach for Data Cryptography, International Journal of Computer Science and Mobile Computing, vol. 8, issue 9, pp. 30-48, 2019.

[32] Ziad Alqadi, Ahmad Sharadqh, Naseem Asad, Ismail Shayeb, Jamil Al-Azzeh, Belal Ayyoub, A highly secure method of secret message encoding, International Journal of Research in Advanced Engineering and Technology, vol. 5, issue 3, pp. 82-87, 2019.

[33] Shaza D. Rihan, A h m e d Khalid, Saife Eldin F. Osman, A Performance Comparison of Encryption Algorithms AES and DES, International Journal of Engineering Research \& Technology (IJERT), vol. 4, issue 12, pp. 151-154, 2015. 